\title{
EFFICACY OF CIPROFLOXACIN AND TETRACYCLINE IN THE MANAGEMENT OF CHRONIC FOLLICULITIS OF LEGS - A COMPARATIVE STUDY
}

\author{
R. Rama1 ${ }^{1}$ G. Suryanarayana ${ }^{2}$, P. Guru Prasad ${ }^{3}$, P. Anila Sunandini' ${ }^{4}$ B. V. Ramachandra ${ }^{5}$, S. Amrutha Bindu ${ }^{6}$, Shaik Asha ${ }^{7}$
}

${ }^{1}$ Assistant professor, Department of DVL, Andhra Medical College, Visakhapatnam. ${ }^{2}$ Assistant professor, Department of DVL, Andhra Medical College, Visakhapatnam. ${ }_{3}^{3}$ Associate professor, Department of DVL, Andhra Medical College, Visakhapatnam. 4 Professor \& HOD, Department of DVL, Andhra Medical College, Visakhapatnam. 5 Retired Professor, Department of DVL, Andhra Medical College, Visakhapatnam. ${ }^{6}$ Postgraduate, Department of DVL, Andhra Medical College, Visakhapatnam. 7Postgraduate, Department of DVL, Andhra Medical College, Visakhapatnam.

\section{ABSTRACT}

Chronic folliculitis of legs results from the inflammation of the ostium of the hair follicle. Clinically, it is characterised by profuse eruption of follicular papules and pustules with the hair piercing the centre of the lesion. It involves predominantly lower legs and also thighs and forearms in severe cases. Usually asymptomatic, but sometimes associated with pruritus and pain. The lesion resolve without any sequelae. There are no associated systemic disturbances. In majority of cases, coagulase positive Staphylococcus aureus isolated from the lesions. It runs a chronic recurrent and relapsing course and usually resistant to treatment affecting the quality of life of the patient. A number of therapeutic agents have been tried in various studies including Cotrimoxazole, Psoralen with UV-A (PUVA) therapy,(1) Ciprofloxacin,(2,3) Rifampicin, Dapsone,(4) Minocycline(5) with variable success rates. Complete cure is not seen with any antibiotic therapy. The present study was undertaken to compare the efficacy of Ciprofloxacin and Tetracycline in the management of chronic folliculitis of legs.

\section{MATERIALS AND METHODS}

Young adults above 18 years presenting with chronic folliculitis were included in the study. It was a comparative study of 100 patients with chronic folliculitis attending Outpatient Department of Dermatology of KGH affiliated to AMC, Visakhapatnam for a period of 1 year. These patients were divided into 2 groups and the efficacy of Ciprofloxacin and Tetracycline in the management of chronic folliculitis was compared at the end of the study.

\section{RESULTS}

With Ciprofloxacin we reported early and excellent response when compared to Tetracycline. Recurrences were reported in all cases studied. Early recurrences were reported following treatment with Tetracycline when compared to Ciprofloxacin.

\section{CONCLUSIONS}

Clinically best results were obtained with Ciprofloxacin in a dose of $500 \mathrm{mg}$ BD for 2 weeks followed by Tetracycline.

\section{KEYWORDS}

Chronic Folliculitis, Ciprofloxacin.

HOW TO CITE THIS ARTICLE: Rama R, Suryanarayana G, Prasad PG, et al. Efficacy of ciprofloxacin and tetracycline in the manage ment of chronic folliculitis of legs - a comparative study. J. Evolution Med. Dent. Sci. 2016;5(71):5152-5153, DOI: $10.14260 /$ jemds/2016/1168

\section{INTRODUCTION}

Chronic folliculitis results from the inflammation of the ostium of the hair follicle. Clinically characterised by profuse eruption of follicular papules and pustules with the hair piercing the centre of the lesion. Predominantly, it affects the young adult males. Predominantly it involves the lower legs, thighs and forearms in severe cases. Usually asymptomatic, sometimes associated with pruritus and pain. The degree of pruritus ranges from mild-to-severe. Lesions resolve without any sequelae. There are no systemic disturbances except hypergammaglobulinaemia in some. Coagulase positive Staphylococcus aureus isolated from the lesions in the majority of cases. Physical or chemical injury may also precipitate the condition.

Financial or Other, Competing Interest: None.

Submission 27-06-2016, Peer Review 20-08-2016,

Acceptance 27-08-2016, Published 02-09-2016.

Corresponding Author:

Dr. P. Guru Prasad,

Associate Professor,

Department of DVL

Andhra Medical College,

Visakhapatnam, Andhra Pradesh, India.

E-mail: gppatnala@yahoo.co.in

DOI: $10.14260 /$ jemds/2016/1168
The Pustular Dermatitis Atrophicans of the legs described in Lagos in West Africa appears to be a similar condition. It occurs predominantly in males and affects symmetrically the anterior tibial surfaces of the legs, sometimes involving the thighs and forearms. In this condition profuse follicular pustules appear followed by atrophic scars. In chronic cases, treatment of carrier sites of Staphylococci is also necessary.

Treatment of chronic folliculitis of legs is frustrating to both dermatologist and patient, as it runs a chronic recurrent and relapsing course affecting the quality of life of the patient. A number of therapeutic agents have been tried in various studies including Cotrimoxazole, Psoralen with UV-A (PUVA therapy),(1) Ciprofloxacin,(2) Pentoxyphylline,(3) Rifampicin, Dapsone,(4) Minocycline(5) and topical Mupirocin with variable success rates. A successful form of therapy is yet to evolve in this disease.

Combination therapy with antiseptic lotions, long-term systemic antibiotics and topical antibiotics and avoidance of risk factors are essential in controlling the disease. Ciprofloxacin, a fluoroquinolone antimicrobial agent, which is known to be active against a broad-spectrum of gram positive and gram negative bacteria was tried in the treatment of this recalcitrant condition. 
In this study, we compared the clinical efficacy of Ciprofloxacin and Tetracycline and the results were analysed. Complete disappearance of the lesions taken as clinical cure.

\section{MATERIALS AND METHODS}

It is a comparative study of 100 adult patients presenting with chronic folliculitis attending the Outpatient Department of Dermatology, KGH/AMC, Visakhapatnam for a period of 1 year.

\section{Inclusion Criteria}

Adult patients more than 18 years who are not taking any treatment either topical or systemic in the previous 3 months.

\section{Exclusion Criteria}

Children, pregnant women and patients on treatment. Patients were divided into 2 groups $\mathrm{A}$ and $\mathrm{B}$.

Group A: Given Ciprofloxacin 500 mg BD 2 weeks.

Group B: Given Tetracycline 500 mg QID 2 weeks.

Disappearance of lesions taken as complete clinical cure. Clinical improvement recorded at the end of second week by grading the response. Patients were followed up every 4 weeks for a period of 6 months. The response to therapy was graded as follows:

Grade 0: No improvement.

Grade 1: 25\% improvement.

Grade 2: 50\% improvement.

Grade 3: 75\% improvement.

Grade 4: Complete resolution of lesions.

Complete dermatological and systemic examination done. Any associated aggravating factors noted. Routine investigations like Complete blood count, Fasting blood sugar, Post-prandial blood sugar, Renal function tests, Liver function tests and Urine examination done. Smear from the lesions examined under direct microscope after gram stain. Pus from the lesion sent for culture and antibiotic sensitivity. Strains were tested for the production of coagulase to know whether the strains were pathogenic or non-pathogenic. In some cases, phage typing also done.

All the organisms isolated from the lesions were tested for antibiotic sensitivity by standard disc diffusion technique with the following concentrations of antibiotics per disc.

- Penicillin - 10 units

- Tetracycline - $30 \mathrm{mcg}$

- Erythromycin - $15 \mathrm{mcg}$

- Ciprofloxacin - $10 \mathrm{mcg}$

- Cloxacillin - $10 \mathrm{mcg}$

- Methicillin - $5 \mathrm{mcg}$

- Lincomycin - $10 \mathrm{mcg}$

- Cephalexin - $30 \mathrm{mcg}$

Coagulase test done to know whether the strains are coagulase positive or negative.

\section{RESULTS}

In all cases, all investigations were normal. Direct microscopic examination of the smear from the lesion after gram stain showed violet coloured cocci in $80 \%$ of cases along with pus cells. In $20 \%$ only pus cells seen, it may be due to technical error.
Culture of the material from the lesions show staphylococci in all cases. All strains are coagulase positive and belong to phage group III and phage type 83A.

All strains show resistance to penicillin in our study. Group A patients who were given Ciprofloxacin $500 \mathrm{mg}$ BD for 2 weeks showed excellent response in $80 \%$ of cases at the end of 2 weeks. Group B patients who were given Tetracycline 500 mg QID for 2 weeks showed response in $60 \%$ of cases at the end of 2 weeks.

In our study recurrences were reported in all patients, but group A patients showed early remission and low recurrence rate when compared to group B. Recurrences are mild with Ciprofloxacin when compared to Tetracycline.

\section{DISCUSSION}

Out of 50 patients in group A, 40 patients (80\%) showed excellent response with Ciprofloxacin at the end of 2 weeks. There was complete resolution of lesions (Grade 4). During follow-up at monthly intervals, recurrences were seen in $40 \%$ of patients. A second course of Ciprofloxacin for another 2 weeks or less cleared the lesions in all of them. The period of remission lasted from 3 to 4 months. Balachandran et al reported response to Ciprofloxacin in $84 \%$ of cases.(2) $\mathrm{He}$ reported average remission period of 44.5 days with Ciprofloxacin. In a study conducted by D Prasad et al, response with Ciprofloxacin was seen in $66.7 \%$ of cases. (3)

D. Prasad et al conducted a study with Ciprofloxacin 500 mg BD along with Pentoxyphylline 400 mg TID for 2 weeks followed by Pentoxyphylline $400 \mathrm{mg}$ TID for another 4 weeks.(3) He reported excellent improvement in $88.3 \%$ of cases. But improvement seen only in $66.7 \%$ of cases treated with Ciprofloxacin alone.

Out of 50 patients in group B, 60\% showed complete resolution of lesions at the end of 2 weeks and the recurrence rate was $50 \%$ and the period of remission was 1 to 2 months. Group A patients had a mild form of the disease during relapse when compared to group B patients.

P. Prasad et al reported $50 \%$ clearance at the end of 2 weeks with Minocycline in a dose of $100 \mathrm{mg}$ once daily one hour before food for 21 days; $25 \%$ showed a mild clinical recurrence.(5)

\section{CONCLUSION}

In our study we found that Ciprofloxacin is more effective than Tetracycline in controlling the disease and it could be used as a first line drug in the treatment of chronic folliculitis of legs, However, it should be confirmed by performing large scale multicentre studies with long-term follow-up.

\section{REFERENCES}

1. Shenoy K, Srinivas CR, Sharma S, et al. Efficacy of cotrimoxazole \& PUVA for the management of chronic folliculitis of legs. IJDVL 1990;56(3):223-5.

2. Balachandran C, Malpani S, Srinivas CR. Ciprofloxacin therapy in chronic folliculitis of legs. IJDVL 1995;61(4):212-3.

3. Prasad D, Saini R, Negi KS. Pentoxyphylline \& ciprofloxacin in chronic folliculitis of legs. IJDVL 1997;63(1):9-10.

4. Prasad PV. Rifampicin \& dapsone in superficial pustular folliculitis. IJDVL 1996;62(1):16-8.

5. Prasad PV. Minocycline in chronic folliculitis of legs. Indian J Dermatol Venereol Leprol 1996;62(5):334. 